\title{
Live Tracking System
}

\author{
Sagar Karkare \\ Dept of Electronics and Telecommunication \\ Rajiv Gandhi Institute of Technology \\ (University of Mumbai) Mumbai, India \\ Pranali Rokade \\ Dept of Electronics and Telecommunication \\ Rajiv Gandhi Institute of Technology \\ (University of Mumbai) Mumbai, India
}

\author{
Ashwini Andhale \\ Dept of Electronics and Telecommunication \\ Rajiv Gandhi Institute of Technology \\ (University of Mumbai) Mumbai, India \\ Santosh Bansode \\ Dept of Electronics and Telecommunication \\ Rajiv Gandhi Institute of Technology \\ (University of Mumbai) Mumbai, India
}

\author{
Ankur Ganorkar \\ Dept of Electronics and Telecommunication \\ Rajiv Gandhi Institute of Technology \\ (University of Mumbai) Mumbai, India
}

\begin{abstract}
Live tracking system means that a GPS tracking device that sends it location to the end user at a consistently high frequency. It offers users real-time location updates, every few seconds. GPS Tracking devices communicate their positions by taking the signals. This system can be used for safety and security purpose by tracking the live location of children's or loved ones.

The days are gone when one of the two parents will sit at home to take care of the children and one earns. Now time has come for both the parents to work; in such scenario the security of children is very important. The numbers of users have android phone equipped with Global Positioning System which can be used efficiently for security and protection purpose. The application is manly developed for a toddler who has many activities to do while in school or outside the home.

Child tracking system is an app that can track and monitor the child location. The aim of the project is to create a system to allow the parents to keep track of their kids when their child is out of their view. However, with the child tracking system the parent can track and monitor their child location in just a simple app when the parent is in office. The Live Tracking System is the multi-purpose safety system since it can be used for any safety purpose tracking which will work in android platform.
\end{abstract}

Keywords:- GPS, Tracking, Android

\section{INTRODUCTION}

Now a days, the use of Android based Smartphone's is increasing day by day. As these Smartphone's are providing various unique facilities, more and more people are getting attracted to them. Thus here, including best possible features and resources has become the need of the day. Moreover, GPS there by device which offers an outstanding services of getting respective location. This facility is helpful in developing application which can track locations of every activity of children while is outside the home. In child tracking system parents are able to trace the activities of their children's through Android Smartphone. The activities such as real time location (on Google Maps).The project is designed to be used by parents and aimed to help locating their children's on the go activity.

In this work, GPS is used along with one of the basic service of a smart phone is SMS. An application at the parent side will allow parents to send a location request to a child side then retrieve the location from the request reply and shows it on a map. On the other hand, the tracing device at the child's side gathers the necessary information such as GPS coordinates which are in the form of latitude and longitude. And time are gathered and sent to the parent smart phone that's pre- registered on the application. The communication between the parent side and the child applications is done using Short Message Service (SMS). SMS offers the system unique features. It will allow the system to work without the need of internet connection thus allows the application to be implemented on smart phones that don't support GPRS. The system sends the location of child's smart phone to parent's smart phone when the parent wishes to check on the child.

\subsection{Background of Study}

We cannot predict or determine what will happen next to us in next seconds. This is the scenario where we cannot acknowledge of when will be targeted, but we can take precaution \& prevent it from happening with us or with our loved ones. Especially our children's are not matured enough to take care of them. So as being a responsible parent, their greatest concern is their children's security. Now a day's most of the parents are working to earn money to sustain a family. Due to the busy working schedule of parents it is not possible for them to always stay beside their children's. Parents will start to feel anxious about their Childs safety \& current status every second because they cannot see what their child is doing currently or what affairs will happen on them.

\subsection{Problem Statement \\ - Less availability of child safety devices with minimum cost}

\section{The parent is hardly to keep a watch on their} child without the use of technology,

especially when the child is in the outdoor. 
$>\quad$ Also the devices available are costlier which is not affordable for middle class parents.

\section{LITERATURE REVIEW}

This chapter briefly describes the review on existing techniques related with the propose project. In this topic, this literature review will discuss about the tracking system. This literature review is a summary and explanation of the complete and current state of knowledge on limited topics as found in journal articles. In this chapter, only three systems are compared for tracking system. There are a large number of studies of the tracking system in the different areas. However, since the focus of this research is on the child tracking system, these will not be reviewed in detail and will only be referred to as appropriate. Besides, studies are also performed on technologies involved and methodology applies in the proposed system that will act as guidance in practicing the best approach for building the tracking system for child.

\subsection{Technical Paper Review}

\subsubsection{Paper 1:International Research Journal of Engineering \& Technology}

\section{Author name: Prof. Shrinivas Sirdeshpande}

Due to rapid increase in population, there is need for efficient public transportation system. There is increased burden on public transportation like bus just because of population. Therefore remote user needs a smart system which provides real time information of bus. So we proposed a new system which solves the drawback of current public transportation system. So our system handles all the data like current location of bus, management of buses and its schedule.

The real time tracking of bus can be done by our proposed system and this information is then given to remote user who wants to know the real time bus information. Some technologies like GPS (Global Positioning System), Google maps and GPRS (General Packet Radio Service) are used for development purpose. Our system provides web based application, which gives real time location of bus on Google Maps to remote user.

\subsubsection{Paper 2: International journal of advanced research in computer engineering \& technology}

\section{Author name: Prof.M.K. Night}

GPS and GSM based device location and tracking system will provide effective, real time device location, mapping and reporting this information back to monitoring device and improving the level of service provided. A GPS based device tracking system will inform where your device is and where it has been, how long it has been. The system uses geographic position and time information from the Global Positioning Satellites. Currently, mostly the existent tracking systems use techniques of virtual fence known as Geophone which compares the entity position with a predetermined zone or a point of interest, checking if the entity is inside or outside an area. Those techniques do not allow full coverage of the course, making difficult to determine if a truck or another delivery device is travelling in a planned path. Therefore, we need to use an alternative technique that allows continuous monitoring of travels, obtaining information of probable deviations or even emergency situations.

\subsubsection{Paper 3: International Journal of Science and Research (IJSR)}

\section{Author name: Miss. Poonam Sather}

The paper describe a practical model for routing and tracking of mobile devices in a large area outdoor environment based on the Global positioning system (GPS) and Global system for mobile communication (GSM). The supporting device GPS continuously move with the car and will calculate the co-ordinates of each position and when required by the owner it can be communicated with the help of GSM modem which is installed in both Transmitter and receiver section. GSM modem is controlled by a 32 bit ARM7 LPC2148. The device will collect position to supervised center by the SMS (Short Message Service) or GPRS (General Package radio service) and which can be located in the Google Earth and so the current position of the car can be known

\subsubsection{Paper 4: International journal of information system and engineering}

\section{Author name: Mr. Joshua Samuel}

Location based services has enable people to locate and track the location of other people, objects, machine, devices and resources, from the comfort of their home as long as they have the required gadget such as smart phone, PDA's, and others (Abuse, et al, 2004). Requesting location sensitive information is usually initiated by a user called the client or network provider. Most application today use Global Positioning System (GPS) provide location information; for example social network site like Facebook allow users to share their location with friends and family, another common example are application that allow users retrieve weather forecast data based on their current location.

With the numerous benefits emanating from the use of location-based service, there is however issues that bothers on the privacy of user; hence there is need for proper government regulations. The purpose of this project to develop a tracking / monitoring Android application (mobile) using object GPS devices to ascertain its current location, and previous location at specified intervals, this system unlike previous tracking system will give user the ability to create bookmark of current location and ability to route back to that location from anywhere using Google Maps API's in case they can't remember the prices location. Chandra, Jain and Qadeer used a simple web server approach along with SMS to solve the problem. It was implemented for JAVA enabled mobile devices equipped with GPS receptor. A client can either send his location to other clients directly by SMS or share it by sending it to the web server's database via internet. Clients can view the locations on the Google maps. The aim of this application is 
to enables the user to share his location with their friends or even who uses the same soelution.

\section{PROPOSED WORK}

\section{A. Requirements}

This work is designed for parents and children. Both must have GPS and GSM services enabled devices i.e. smart phone at the parent side and the tracking module at child side that supports GPS and SMS as a minimum. SMS is a basic service on any smart phones but GPS can be found on new smart phones. The application is mostly to be used by parents to track down the child's location. In a later phase for implementation purposes, the system will be developed using Android App. The Android App to be used is a digital dashboard where a graphic interface can be build.

The main reason why the Android OS was chosen for the implementation of this work is to target more users. Statistics shows that the market share for the Android OS is 48.8. This makes it the highest over others Smartphone's operating systems currently in the market.

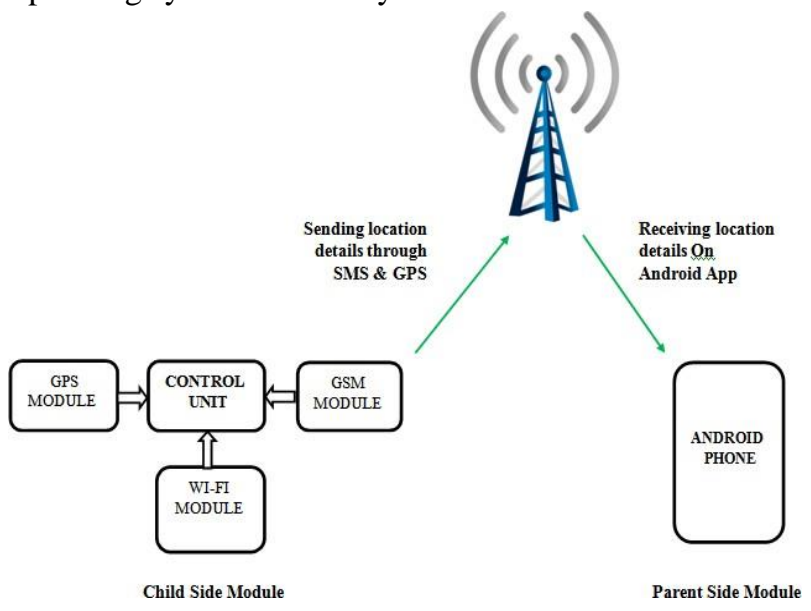

Fig.: Proposed System

\section{B. $\quad$ Application Architecture}

We propose a solution to solve the problem based mainly on GPS and GSM technologies. It takes advantage of the two main rich features that is offered in Android platforms nowadays. Those features are location services, mainly GPS, and basic telephony services, mainly SMS. The system proposed is based on a simple idea that is the use of SMS for communicating between the parties involved, parent and child. It is designed in a simple way so that it will involve few elements and less user interaction. This way it will result in a system that is simple and easy to implement and use, making it more user-friendly. The architecture of the system proposed, consists of two sides. First is the parent side which acts as a server for the system though it is not actually a server. It is basically an Android phone owned by the parent of the child for tracking and monitoring. The parent's side uses SMS for communicating and receiving the alert messages and location co-ordinates from child module and maps to view the location of the child on a map. Thus, it requires telephony and internet services to be enabled in the parent's phone for the system to function

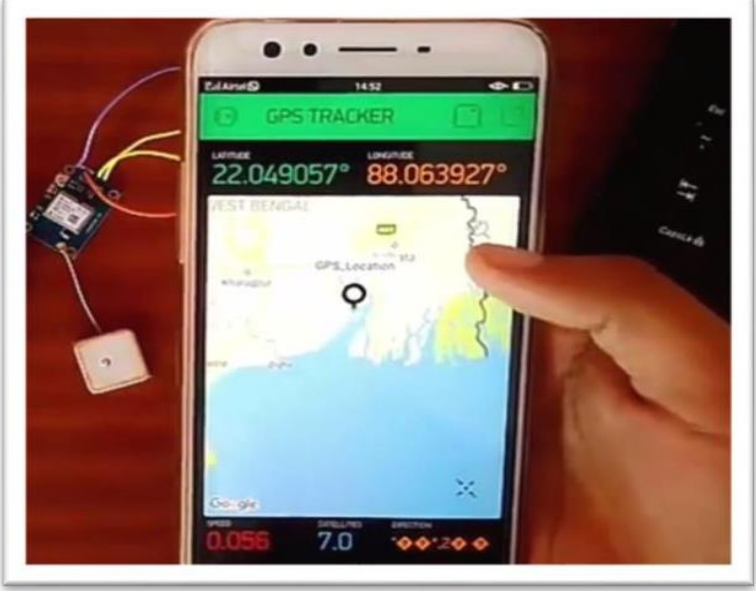

Fig.: Parent Side

The Second is the child side which acts as a client for the system. The child side is a traceable module consists of GSM and GPS devices. The child side uses SMS for communicating with the parent side and location services, GPS or Network, to get the location of the child in form of coordinates. On the child side, telephony and location services must be enabled and up running on the child side for the system to work. Where else the parent side might need internet connectivity only for the map to show.

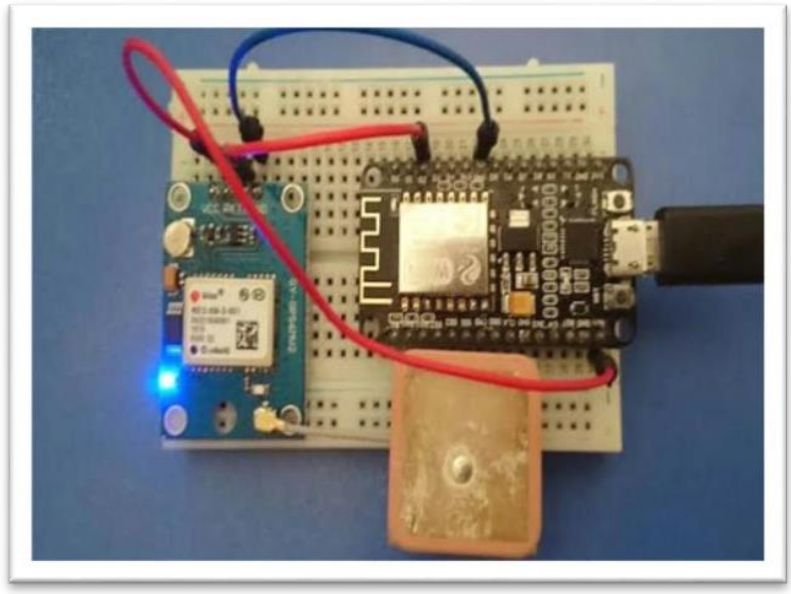

Fig.: Child Side Module

On the parent side, there is an Android phone with an application for monitoring child location. The Android App has a digital dashboard where graphic interface can be build according to requirements. After creating required interface for tracking application on an Android App, child module can be traced continuously on the map. The Android App to be used allows number of user to access the created tracking interface by logging into the interface. On the child side, the traceable module consists of GSM and GPS devices. The child side uses SMS for communicating with the parent side and location services, GPS or Network, to get the location of the child in form of coordinates such as longitude and latitude. A parent will use the interface to track the location of child. An Android app service runs in the background continuously so that parent can see the child's real time location at any time and the child module keeps on sending 
the location coordinates i.e. latitude and longitude through SMS as soon as the child reaches to the Pre-defined check points as a confirmation. The design of this proposed solution offers many advantages over many exiting solutions.

First, the application operates automatically as soon as child reaches to the pre-defined check points without the need for user interaction at the child side. This is considered as a big advantage for the system. Another advantage is that the system uses SMS for data transfer thus eliminating the need for internet connectivity. The system will only require location services and telephony connectivity. This is suitable for situations where the users might not have internet access. The third advantage this solution offers is that it can perfectly function indoors as well. It is done by using both GPS and Network provider for location determination. The application will always get coordinates from both, compare them and use the most accurate.

In cases where there is no GPS satellite signal received, for example indoors, the application will use the only other source available which is the Network provider. It should be noted that Network provider location detection is based on the Cellular ID. Lastly, the system uses a master slave relation between parent and child sides where the parent controls all the functions of the system and the child has very little control over the system.

\section{CONCLUSION}

The proposed system is designed to aid locating the live location of children's who are minors for their safety purpose. The architecture of system is mainly built on two basic features i.e. Global Positioning System(GPS) and SMS services used to track the location and to send the messages to the user. The tracked location is decided by the tracked parameters such as latitude and longitude. Using this parameter the user can verify the location by putting received values on the Google of latitude and longitude. The advantage of the proposed system is here the user get one more option to track the device i.e. Android Application which also include the map option for user to observe.

\section{REFERENCES}

[1] (Android \& Google, 2016) Android, \& Google. (2016). Android Studio Features Android Studio. Android. Retrieved from https://developer.android.com/studio/features.html

[2] (El-rabbany, 2006) El-rabbany, A. (2006). Introduction to GPS: The Global Position System. Navtech GPS Part 1221. https://doi.org/10.2493/jispe.72.285

[3] FiLIP, 2016. FiLIP - THE WORLD'S FIRST SMART LOCATOR AND PHONE FOR KIDS. [Online] Available at http://www.myfilip.com/ [Accessed 2 Nov.2017].

[4] (Gatwick \& Ahmad, 2011)Gatwick P., \& Ahmad, K. (2011). App Inventor for Android with Studio-based tracking system using GPS and GSM modem. In 2013 IEEE Conference on Open Systems, ICOS $2013 \quad$ (pp. https://doi.org/10.1109/ICOS.2013.6735054

[5] (Rycroft, 1997) Rycroft, M. J. (1997). Understanding GPS. Principles and applications. Journal of Atmospheric and SolarTerrestrial Physics, 59(5), 598-599. https://doi.org/10.1016/S1364-6826 (97)83337-8

[6] (Sarjana \& Ii, 2012) Sarjana, P., \& Ii, M. (2012). GSM \& GPS BASED SCHOOL KIDS TRACKING SYSTEM NG WOON CEA This Report Is Submitted In Partial Fulfillment of Requirements for the Award of Bachelor .Degree of Electronic Engineering ( Industrial Electronic) With Honors Faculty of Electronic Engineering.

[7] (Salihoglu \& Widom, 2013) Salihoglu, S., \& Widom, J. (2013). Gps. Proceedings of the $25^{\text {th }}$ International Conference on Scientific and Statistical Database ManagementSS 